\title{
Maxwell's demon opens new doors
}

\author{
Adela Marian and Bretislav Friedrich \\ Fritz-Haber-Institut der Max-Planck-Gesellschaft, Berlin, Germany
}

(Dated: July 25, 2008)

Maxwell's demon's back - and he works for food. In an almost literal realization of Maxwell's thought experiment, a group of physicists at the University of Oregon demonstrated a one-way gate, which lets through atoms incident from one side but sends them back when they come from the other side. As a result, the atoms crowd only one side of the gate. In order to carry on with his gate-keeping job, the demon picks up foodstuff - in the form of information about the internal state of the impinging atoms. As Thorn et al. [? ] have shown, providing a menu with a choice between merely two internal states of the atoms is enough for the demon to subsist. With the gate employed to partition an atom trap, the demon increases the atoms' density on one side of the partition - at the expense of the density on the other side.

The one-way gate is based on the idea that the character of the interaction between an atom and a laser beam depends on the laser beam's detuning with respect to a specific transition within the atom. If the laser frequency is lower than the transition frequency (red detuning), the laser beam exerts an attractive force on the atoms, pulling them towards the region of maximum laser intensity. If, on the other hand, the laser frequency is higher than the transition frequency (blue detuning), the beam repels the atoms, pushing them away from the beam's focus.

Two focused laser beams are needed in order to experimentally realize the unidirectional gate, as shown in Fig. ??. The first beam (Beam 1) is tuned between the $F=1 \rightarrow F^{\prime}$ and $F=2 \rightarrow F^{\prime}$ electronic transitions, which results in an attractive well for the atoms in the $F=1$ state (shown in red) and a repulsive barrier for the atoms in the $F=2$ state (shown in blue). The second beam (Beam 2 ) is resonant with the $F=1 \rightarrow F^{\prime}=2$ repumping transition and promotes all the atoms within the electronic ground state into the $F=2$ state.

But how does the one-way action of the barrier come about? Suppose an atom in the $F=1$ state starts out on the left side of the barrier, as indicated by the matching red arrow in Fig. ??a. Beam 1 is attractive for this state, so the atom will cross it. However, once within the reach of Beam 2, the atom will be promoted to the $F=2$ state. This is depicted by the blue arrow emerging on the right side of the barrier. Since for atoms in the $F=2$ state, Beam 1 is repulsive, an atom in the $F=2$ state will be forced to stay put on the right side of the barrier. If, on the other hand, the $F=1$ atom starts out on the right side of the barrier, it will be immediately promoted to the $F=2$ state by the repumping laser (Beam 2), and subsequently reflected back by the repulsive barrier due to Beam 1. As a result, Beams 1 and 2 act on atoms in the $F=1$ state as a wall which is permeable to the ones impinging from the left but reflecting to the ones coming from the right, see Fig. ??b.

One would naively expect the wall to be completely reflective for atoms in the $F=2$ initial state, no matter what side of the barrier they come from. But the measurements carried out by Thorn et al. tell a different tale. For atoms coming from the left side, they observe at first reflection by the barrier, but at later times they can clearly see transmission through the barrier. This is due to the particular choice of the asymmetry of the detuning in the experiment (the blue detuning is less than the red detuning, see Fig. ??), leading to optical pumping into the transmitting $F=1$ ground state.

The one-way wall of light is particularly important as a new tool to spatially compress an atomic sample and thus further cool the atoms [? ]. Barriers can in principle be designed for a variety of atoms and molecules. In contrast, the commonly used method of laser cooling requires a two-level cycling transition and is thus restricted to a narrow class of atoms, best represented by the alkalis. In the case 
of laser cooling, heat dissipation occurs via momentum transfer from the photons in the laser beams to the atoms in the vapour, and relies on multiple photon scattering. In the case of the one-way barrier, scattering only a single photon is sufficient to obtain a comparable dissipation [? ].

Another method employed to obtain ultra cold atoms is evaporative cooling, which has been instrumental in reaching the quantum degenerate regime in gases (one of whose manifestations is a Bose-Einstein condensate). Because evaporative cooling critically depends on the rate of elastic collisions among the atoms, the enhanced density afforded by the one-way barrier could be used to trigger evaporative cooling.

[] J.J. Thorn, E.A. Schoene, T. Li, and D.A. Steck, "Experimental realization of an optical one-way barrier for neutral atoms," Phys. Rev. Lett. 100, 240407 (2008).

[] M.G. Raizen, A.M. Dudarev, Q. Niu, and N.J. Fisch, "Compression of atomic phase space using an asymmetric one-way barrier," Phys. Rev. Lett. 94, 053003 (2005).

[] G.N. Price, S.T. Bannerman, K. Viering, E. Narevicius, and M.G. Raizen, "Single-photon atomic cooling," Phys. Rev. Lett. 100, 093004 (2008). 
Beam 1 Beam 2
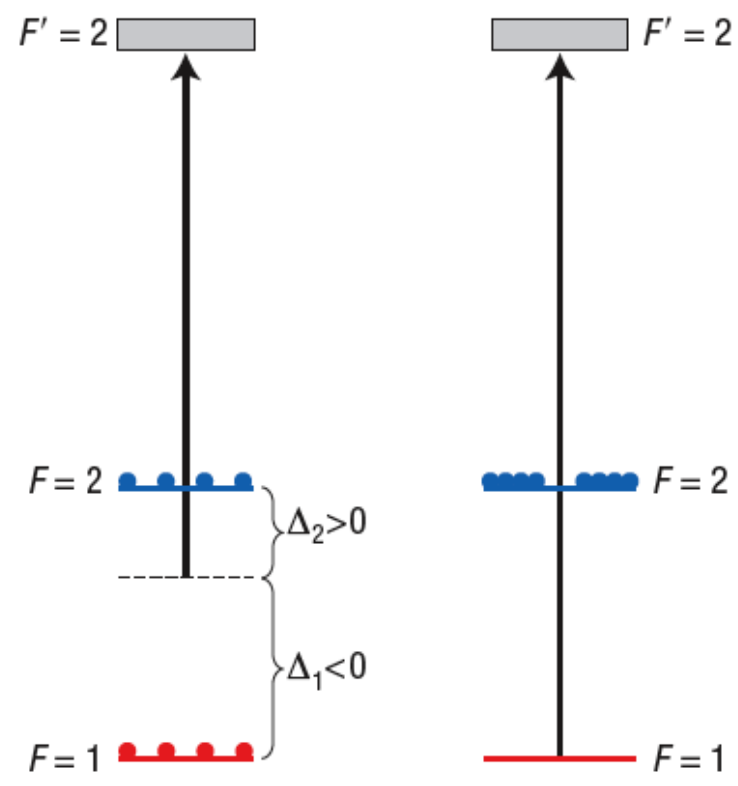

FIG. 1: Energy levels and optical transitions employed in the one-way barrier experiment with rubidium atoms of Thorn et al. [? ]. While the $F=1$ (red) and $F=2$ (blue) hyperfine states are from within the electronic ground state $\left(5^{2} S_{1 / 2}\right)$ manifold, the $F^{\prime}=2$ state belongs to the electronically excited state $\left(5^{2} P_{3 / 2}\right)$. The detuning of the barrier beam (Beam 1) is negative with respect to the $F=1 \rightarrow F^{\prime}=2$ transition $\left(\Delta_{1}<0\right)$ and positive with respect to the $F=2 \rightarrow F^{\prime}=2$ transition $\left(\Delta_{2}>0\right)$. The repump beam (Beam 2$)$ is resonant with the $F=1 \rightarrow F^{\prime}=2$ transition. The red and blue circles show schematically the populations of the $F=1$ and $F=2$ levels that result from the interaction of the atoms with Beams 1 and 2. See text. 


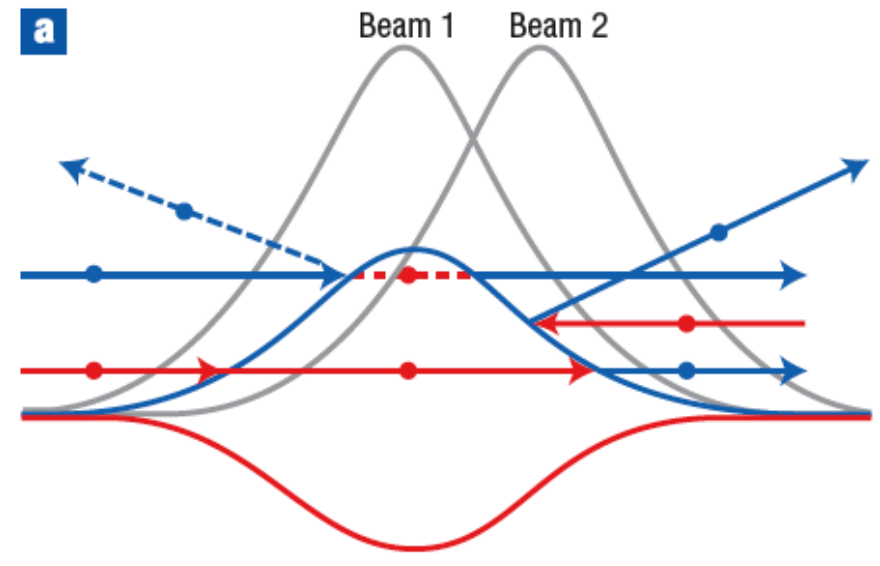

b

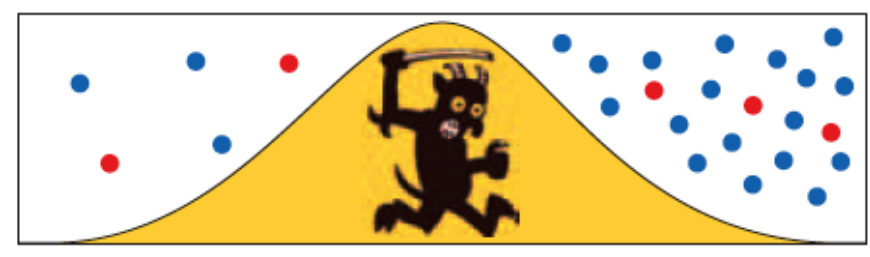

FIG. 2: (a) The potential well (red) and potential barrier (blue) created by the barrier beam (Beam 1, grey). The repump beam (Beam 2, grey) transfers population on the right side of the barrier from the transmitting $F=1$ state to the reflecting $F=2$ state. The typical trajectories of atoms in the $F=1$ and $F=2$ states are shown by matching color-coded arrows. Dashed lines indicate a partial transnission or reflection. (b) Schematic of the atom populations left and right of the one-way barrier. The barrier partitions an atom trap which spatially confines the atoms. Note that most of the atoms accumulated on the right are in the $F=2$ state. See text. 